\title{
Algunos aspectos de estructuración de los programas de prevención en farmacodependencias
}

Some aspects of structuring the prevention on drug abuse programs

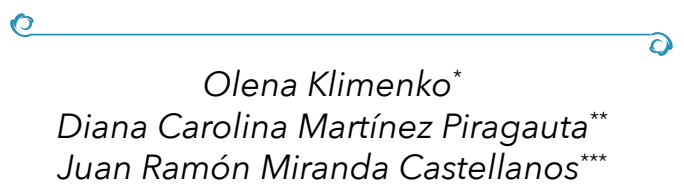

Recibido: 10.06.2018 • Arbitrado: 12.07.2018•

Aprobado: 25.08.2018

Psicóloga, Magister en Ciencias Sociales, Doctoranda en Psicopedagogía, Docente Institución Universitaria de Envigado, Escuela de postgrados de la Universidad Católica Luis Amigó, olenak45@gmail. com, ORCID: 0000-0002-8411-1263

* Psicóloga, Especialista en Psicología Clínica y Autoeficacia Personal de la Universidad El Bosque, Especialista en Adicciones de la Universidad Católica Luis Amigó, ps.dianamartinez@ yahoo.com

*** Médico de la Universidad de Cartagena, Especialista en Adicciones de la Universidad Católica Luis Amigó, juanramiranda2510@hotmail.es

\section{Resumen}

El artículo presenta resultados de una revisión sistemática sobre el tema de estructuración de programas de prevención en farmacodependencias. Se empleó la metodología de enfoque cualitativo, nivel descriptivo y método bibliográfico, se seleccionaron artículos y documentos científicos publicados entre los años 2005-2017 en idiomas inglés y español, encontrados en bases de datos como Science Direct, Redalyc, Scielo, Proquest y Ebsco host, se utilizó el método de análisis intra e intertextual. Los resultados de la revisión permiten identificar algunos aspectos clave en el proceso de planeación, estructuración y ejecución de los programas preventivos en el consumo de sustancias.

Palabras clave: revisión sistemática, prevención, sustancias psicoactivas, consumo, intervención 


\section{Abstract}

This research project aims to perform a systematic review of the structuring, planning and evaluation of prevention programs in drug dependencies, which allow to know in depth the requirements and the development of effective programs. For this, articles and scientific documents published between 2005-2017 in English and Spanish were selected, found in databases such as; Science Direct, Redalyc, Scielo, Proquest and Ebsco host, was used the inside and between textual analysis method. The results of the review allow to identify some key aspects in the process of planning, structuring and executing the preventive programs in the consumption of substances.

Keywords: systematic review, prevention, psychoactive substances, consumption, intervention

\section{Introducción}

El consumo de sustancias psicoactivas a lo largo de la historia ha venido tomando cada vez mayor fuerza como un fenómeno social que se ha caracterizado por la alteración de la funcionalidad del sujeto dentro de su accionar como miembro de un sistema. La percepción del consumo como una enfermedad y problemática de salud pública, ha venido desarrollándose desde los años 50's, ya que el consumo ha tenido múltiples cambios, considerando que desde épocas ancestrales el ritual de la alteración de la conciencia por medio de influencia química ha sido común en distintas sociedades y contextos. La situación problema ha surgido desde que el uso de sustancias psicoactivas ha salido del contexto de creencias y usos culturalmente limitados a una población reducida, expandiendo su empleo a la población general y privándolo de un contexto ritual y significativo, generando problemáticas de adicción y daño a nivel individual y comunitario (Castellanos Obregón y Espinosa Herrera, 2013).

Según el último informe mundial de drogas, se calcula que a nivel mundial más de 250 millones de personas de entre 15 y 64 años han consumido por lo menos una vez en su vida algún tipo de sustancia psicoactiva y los índices de consumo han aumentado de forma tan alarmante que se considera que la población consumidora asimila la población total de países como Francia, Italia y Alemania (UNODC, 2016). Igualmente, los problemas relacionados con el consumo como el VIH, las muertes relacionadas con drogas, la formación de grupos al margen de la ley y el narcotráfico, han aumentado en las últimas décadas, siendo la población de los jóvenes más afectada en diferentes áreas 
como la médica, psicológica, familiar y psicosocial (Ferrel Ortega, Ferrel Ballestas, Alarcon y Delgado Arrieta, 2016). El consumo y abuso de drogas está asociado, también, con enfermedades de índole cardiaca, digestiva, neuronal y respiratoria, contribuyendo al aumentos de problemáticas de salud pública (Correa Muñoz y Pérez Gómez, 2013).

Otro de los temas importantes en relación al consumo está relacionado con los adolescentes y jóvenes, quienes representan la población más vulnerable y de mayor consumo de sustancias debido a múltiples factores de riesgo asociados con esta edad evolutiva. Los adolescentes inician el consumo cada vez más temprano, siendo el uso del cannabis el más popular dentro de esta población. Su consumo no solo perdura a lo largo de los años sino actúa como puerta de entrada para otras sustancias (Galvan, Guerrero Martelo, Torres Oviedo, Torres y Sanchez Caraballo, 2015). Igualmente, la legalización del uso de marihuana medicinal y el porte de dosis personal, pueden ser usados por los jóvenes como una estrategia para buscar aceptación a su conducta de consumo dentro de la sociedad (Sáenz Uribe, 2009).

Algunos estudios sobre el consumo recreativo de sustancias, indican que los adolescentes manejan un imaginario de aceptación positiva frente al consumo, sobre todo de cannabis, subestimando su poder adictivo y repercusiones del consumo, basándose en su representación como sustancia natural de uso medicinal. Lo anterior contribuye a que los jóvenes manejar una tranquilidad y aceptación de su consumo, Convirtiéndolo en un estilo de vida y una elección, sin considerarlo como adicción (Ruiz Olivares, Lucena, Jose Pino, Raya y Herruzo, 2010).

La magnitud de consumo y la continuidad en la detección de problemáticas asociadas a este, han generado la necesidad del establecimiento de estrategias de prevención, llevando a determinar la estructuración de programas de prevención que beneficien el fomento y el mantenimiento de factores protectores que permitan generar una mejor protección en los jóvenes que permita orientarlos en toma de mejores decisiones en cuanto al consumo de sustancias (Cogollo, 2013).

Durante un tiempo se empleó el método de control del narcotráfico y los expendios de drogas como el más efectivo para acabar con la problemática, sin embargo, los estudios han demostrado la importancia de intervención y sobre todo de la prevención relativa a otros factores psicosociales relacionados con la problemática del consumo (Hernández Ramírez, 2015).

La oficina de las naciones unidas contra la droga y el delito ha especificado que la funcionalidad de los programas no nace específicamente de la 
necesidad del abordaje netamente del riesgo del consumo de las sustancias psicoactivas, sino de aquellos factores que se asocian como desencadenante del consumo de sustancias. Factores como el contexto comunitario, la familia, el sistema escolar, el área personal del individuo, nivel socio-económico, entre otros, que se han asociado como factores de riesgo (Hernández Ramírez, 2015).

Los programas de prevención se formulan con el fin de proveer una educación sobre el consumo de todas las sustancias psicoactivas y fortalecer los factores de protección presentes en diferentes ámbitos. Las principales estrategias se basan en los principios de participación activa de los usuarios dentro del desarrollo de los programas, otorgándoles un papel activo en la detección y abordaje de los factores de riesgo propios de su contexto. Según Fernández Cruz, Gijón Puerta y Lizarte Simon (2016), la ONU considera varios aspectos para la estructuración de la prevención, como la evaluación del riesgo que cada comunidad conlleva, estrategias basadas en la búsqueda de la reducción del consumo de todas las sustancias, trabajo con la comunidad con el fin de identificar las necesidades y los ejes de la intervención y prevención, y la estructuración de las acciones encaminadas a la disminución y evitación del desarrollo de elementos que propician el inicio de un consumo, entre otros.

Los estudios relacionados con el fenómeno de consumo evidencian la importancia de abordaje de los factores protectores y de riesgo con fines de prevención de consumo, tales como la intervención con la familia, la comunidad, el área educativa, los espacios comunitarios de libre desarrollo que brinden un uso del tiempo libre sano y de provecho, al igual como atención a nivel individual orientada a fomentar mejores herramientas para la vida. Estos aspectos son los que recogen los programas de prevención, donde se busca llegar a todos aquellos involucrados en el problemática y que son actores de cambio, permitiendo fortalecer habilidades e impulsar un consumo responsable (Muñoz Ortega et al., 2012).

Los estudios en cuanto al análisis de la problemática a nivel nacional, han sido cada vez más preocupantes y el tema de la prevención es cada vez más común dentro de los planes de desarrollo departamentales y municipales, ya que el consumo de sustancias afecta a la comunidad en general y es obligación de las autoridades nacionales y del estado brindar las estrategias que permitan disminuir los riesgos y, por ende, disminuir los casos de desarrollo de adición a las sustancias tanto légales como ilegales (Moreno, Posada y Jaramillo, 2012).

La estructuración de programas de prevención provee a la comunidad sanitaria una estructuración de las necesidades ubicadas dentro de su comunidad 
en relación al consumo de sustancias psicoactivas, determinando una ruta para el manejo de las acciones que permitan brindar la atención preventiva necesaria para los casos en colectivo y de esta forma orientar y fomentar una cultura sin riesgos y empoderada en la prevención (Cepeda Diaz, Pezzano de Vengoechea y Racedo de Barranco, 1990).

En Colombia, debido las cifras de cada vez creciente consumo de sustancias, y sobre todo en la población adolescente, se requiere generar mayores avances en cuanto al tema de prevención de las farmacodependencias, brindando programas de fácil implantación y durabilidad, que se trabajen bajo el concepto de calidad con el desarrollo de sistemas de evaluación que permitan verificar que los programas brindan lo que ofertan y reflejan una disminución del riesgo del consumo (ODC, 2017).

Es necesario empoderar distintos agentes comunitarios en cuanto a esfuerzos en la selección, ejecución y evaluación de estos programas, lo que genera la necesidad de inicialmente conocer todos los determinantes y factores que a lo largo del tiempo se han utilizado para la estructuración de este tipo de programas (Becoña Iglesias, 2001).

\section{Método}

El proceso de revisión se enmarca en un estudio de enfoque cualitativo, nivel descriptivo y método bibliográfico, clasificándose como una revisión sistemática siguiendo el modelo propuesto por Peticrew y Roberts (2008), orientada al tema de la estructuración, planteamiento y evaluación de programas de prevención en farmacodependencias.

\section{Criterios de búsqueda}

Se realizó la búsqueda en las bases de datos como Science Direct, Redalyc, Proquest, Scielo, APA PsycARTICLES y EBSCO Host utilizando operadores lógicos. Como buscadores se utilizaron las palabras clave consumo, sustancias psicoactivas, prevención del consumo de sustancias, programa, en su respetivo idioma español e inglés.

\section{Criterios de inclusión}

Artículos de investigación nacional o internacional relacionados con el tema de programas de prevención para el consumo de sustancias psicoactivas, en idioma español o inglés, realizados por profesionales de la psicología, 
psiquiatras, trabajadores sociales $\mathrm{u}$ otros profesionales del área de la salud $\mathrm{y}$ las ciencias sociales.

\section{Procedimiento}

El primer filtro de evaluación se efectuó mediante una revisión detallada del título y resumen de los artículos seleccionados, seguido por el análisis de los objetivos, método, resultados y discusión de los mismos, con el fin de asegurar la calidad y el aporte al tema de investigación, descartando para el análisis los que no cumplían con el criterio, siendo incluidos, sin embargo, en las matrices de información sobre los datos del proceso. Se empleó una lista de chequeo de análisis de artículos, calificado con media o alta calidad para la inclusión en el análisis final (Peticrew y Roberts, 2008). La búsqueda arrojo un total de 371 documentos, con la mayor participación de bases de datos de Proquest y EbscoHost. Dentro de la primera revisión se eliminó más del $68 \%$ de los textos por la falta de cumplimiento de los criterios de inclusión. Del total de los resultados se manejó un total de 56 documentos para el análisis final.

Los datos obtenidos se plasmaron en tablas y matrices comparativas para documentar cada paso y mostrar los resultados de la búsqueda de forma organizada y resumida para facilitar el proceso de revisión.

\section{Consideraciones éticas}

En la construcción del texto se tuvo en cuenta la reglamentación para derechos de autor y normas de citación (APA, 2006).

\section{Desarrollo del tema}

Evolución en la orientación de las estrategias de prevención en torno al consumo de sustancias

La prevención en las farmacodependencias y las conductas de abuso es la estrategia más utilizada, desde la salud pública, para abordar la problemática del consumo hoy en día. Considerando la presencia de riesgos tanto para el inicio del consumo, como para el desarrollo de adicción, los programas de prevención permiten orientar y brindar la información necesaria para que el individuo adopte medidas de prevención. Entre estas se puede distinguir programas que parten de la relación entre el consumo y los factores detectados como riesgo y/o de protección; y las que parten de modelos explicativos de base para su respectiva estructuración. 
La prevención de las farmacodependencias se entiende como un proceso activo de implementación de iniciativas que buscan modificar y mejorar la formación integral y la calidad de vida de los individuos que están en riesgo, en la que se busca aumentar el autocontrol, la toma de decisiones, el proyecto de vida y la resistencia al consumo (Becoña, 2002).

La prevención se centra, principalmente, en el trabajo con la población que puede estar cercana a los factores de riesgo pero que aún no consume y la población que ya se encuentra en un consumo inicial o lo ha llevado a cabo con fines recreativos, lo cual aún no genera una alteración y no se ven implicados daños fisiológicos y cognitivos (Becoña, 2002).

A partir de los años 60, desde que el uso de las drogas se convirtió en más que un objetivo recreativo y se visualizaron los daños, los programas de prevención dieron sus primeras apariciones implementando las estrategias basadas en los conocimientos netos de la población sobre el consumo, siendo su principal objetivo proporcionar información sobre el uso de las drogas y sus efectos a toda la población en general. Estos programas tenían un enfoque más médico que psicológico y, a lo largo de los años, no han demostrado su eficiencia (Castaño, 2006).

Gradualmente, a partir de la evaluación de las estrategias implementadas, terminando los 70 e iniciando los años 80 , los programas de prevención se orientaron, cada vez más, hacia estrategias dirigidas a aspectos psicosociales, centrándose cada vez más en las características específicas de las poblaciones concretas (Castaño, 2006). Estas estrategias apuntaban al trabajo con los factores de riesgo tanto a nivel personal, como social, considerando que el consumo se da en un determinado ambiente que pueda tanto facilitarlo, como prevenirlo, partiendo de diferentes representaciones y aceptación social de consumo (Martínez, 2006).

Hacia el inicio de la década de los 90 surge un enfoque de prevención basado en al fomento de habilidades generales que se denominaron como habilidades para la vida (Martínez, 2006), partiendo desde el punto de vista de que una mayor fortaleza a nivel personal permite afrontar y manejar mejor las influencias sociales, ayudando a la persona escoger un camino diferente al consumo. En esta dirección uno de los programas de interés fue de Botvin et al. (1997), implementado en el ambiente escolar y orientado a reforzar en los adolescentes las competencias sociales con el fin de mejorar su resistencia a las influencias ambientales.

Actualmente, prevalece una tendencia de prevención basada en evidencia (Martínez, 2006). Este tipo de prevención parte de la consideración de evi- 
dencia sobre factores que inciden y/o predisponen hacia el consumo, construyendo programas que permiten incidir en diversos factores de riesgo al igual como fomentar los factores protectores presentes en la población determinada. La identificación de estos factores puede estar relacionada tanto con las investigaciones previas en las poblaciones semejantes y/o indagaciones previas diagnosticas sobre las características específicas de la población objeto. Este tipo de prevención involucra de forma activa la población permitiendo, de esta forma, partir en la construcción de programas orientadas a las necesidades reales de personas y comunidades, al igual como trabaja en paralelo con procesos investigativos interdisciplinarios. NIDA (2004) identifica los principios en los cuales deben basarse los programas de prevención basada en videncia, entre los culés se pueden destacar: orientación tanto a la disminución de factores de riesgo, como fomento de factores de protección concretos, presentes en comunidades a los cuales se dirigen los programas; amplio rango de acción en cuanto a diferente tipo de drogas tanto legales como ilegales; orientarse a la prevención de consumo en edades muy tempranas, al igual como a las familias, apuntando al mejoramiento de dinámicas familiares y pautas de crianza esenciales para un desarrollo sano de los hijos; fomento de resiliencia en los ambientes escolares, apoyo a los procesos escolares de los estudiantes, fortalecimiento de sus habilidades sociales y personales; estrategias orientadas a las comunidades competas por medios de compañías en medios de comunicación, organizaciones religiosas, etc.; una proyección a largo plazo, con repetidas intervenciones, con el fin de logra un efecto esperado; capacitación de personas involucradas como profesores, padres de familia orientada a un mayor empoderamiento de las personas participantes; prevalencia de técnicas y actividades interactivas con el fin de lograr una mayor participación, involucramiento y compromiso.

Igualmente, el enfoque de prevención basada en la evidencia requiere de procesos de seguimiento y evaluación de los programas implementados con el fin de identificar su eficacia real.

La anterior evolución, en cuanto al abordaje de estrategias preventivas, se reflejó, igualmente, en los cambios al respecto de concepción general sobre la organización del proceso preventivo en farmacodependencia. Inicialmente el modelo general de prevención se basó en la definición de tres tipos de prevención: primaria, cuyo objetivo principal es evitar que el problema se presente, se interviene antes del inicio del problema de consumo; secundaria, tiene como objetivo la intervención oportuna sobre el problema cuando ya se ha desarrollado y no se pudo impedir su aparición por medio de la prevención primaria y terciaria, que se aplica con el fin de evitar las complicaciones o recaídas (Caplan, 1980, citado por Becoña, 2002). 
A medida que los factores de riesgo para el consumo se iban incrementando en distintos sectores de la población, creando la necesidad de mejora en cuanto a los programas y el establecimiento de modelos, aparece una nueva clasificación sobre tipos de prevención, propuesta por Gordon (1987, citado por Becoña, 2001). La nueva propuesta de clasificación plantea mejores criterios para la selección de la población, diferenciándolas por las acciones y determinando estrategias centradas en la necesidad. Este modelo las divide en prevención universal, que va dirigida a un grupo poblacional amplio, por ejemplo, adolescentes, y cuyo fin es fomentar en esta población diferente tipo de habilidades que permitan prevenir la emergencia de un posible consumo, como habilidades resilientes, habilidades para la vida, valores y actitudes hacia el consumo, entre otros; selectiva, que se dirige a un subgrupo de la población general que presenta un mayor riesgo que el promedio de sus pares de ser consumidores; y la prevención indicada, que va dirigida a un subgrupo mas concreto, suelen ser ya consumidores que presentan una alteración de comportamiento, por lo tanto, se considera como un grupo de alto riesgo (Becoña, 2002).

Recientemente algunos autores se refieren a un cuarto tipo de prevención, denominada ambiental (Burkhart, 2011). Este tipo de prevención se emplea, sobre todo, en países del norte de Europa, a nivel macro en cuanto a las políticas de control de alcohol y tabaco, al igual como las políticas locales para mejorar el clima en ambientes educativos y contribuir a diversificar los ambientes de diversión. La prevención ambiental está orientada a modificar el contexto físico, económico, social y virtual en el cual las personas toman decisiones al respecto del consumo, lo cual implica considerar, a la hora de construir programas de prevención, los aspectos sociales y económicos a nivel macro, donde se desenvuelve el individuo, pasando por condiciones de su ambiente social más inmediato como comunidad, centros educativos, espacios recreativos y terminando con ambientes inmediatos como la familia (Burkhart, 2011). De esta forma la prevención ambiental se centra en contexto y normas sociales que actúan sobre el consumo de sustancias en diferentes contextos culturales y sociales.

\section{Prevención, programa de prevención y acción preventiva.}

Ministerio de Salud y Protección Social y UNODC (Oficina de las Naciones Unidas contra la Droga y el Delito) (2015) diferencia entre los conceptos de prevención, programa preventivo y acción preventiva. 
Prevención se concibe como un amplio conjunto de medidas orientadas por el propósito de atender el problema de consumo como un fenómeno social, partiendo desde el enfoque de protección social y enfocándose en el abordaje de riesgos psicosociales de comunidades al respecto del consumo de sustancias (UNODC, 2015). Desde esta perspectiva ha surgido el concepto de manejo social del riesgo, que básicamente esta orientado a controlar los riesgos asociados al consumo, reduciendo la vulnerabilidad de las poblaciones especificas hacia el consumo mediante introducción de estrategias de inclusión social y fortalecimiento de redes sociales en diferentes niveles (Ministerio de Protección Social, 2011).

En términos generales, la prevención busca, entre sus objetivos, que el consumo no ocurra, o en caso de que esto no sea posible, que disminuya la edad de su inicio, se limita la cantidad de sustancias consumidas y/o se evitan las consecuencias negativas de este.

El logro de estos objetivos se hace mediante el diseño de diferentes estrategias específicas orientadas a los aspectos particulares relacionados con el tema de consumo, agrupados, a su vez, en ejes temáticos y/o áreas de intervención según características de la población objeto, constituyendo de esta forma programas concretos de intervención. Un programa es el diseño de las actuaciones que se desean implementar para alcanzar la meta deseada, en este caso un programa preventivo, son aquellas actuaciones que tienen el objetivo específico de impedir la aparición de la problemática, buscando que la población afectada genere una conducta preventiva, impidiendo y/o retrasando la aparición de conductas de consumo, de uso y de abuso de sustancias psicoactivas (Becoña, 2002).

En algunas ocasiones los términos se confunden llegando a implementar tomando una base inadecuada para el desarrollo del programa, en primera medida basada en el uso del término prevención, el cual es ya muy genérico. El programa preventivo debe ser específico por medio de la estipulación de unos objetivos concretos y delimitación clara de los grupos objeto de intervención. El análisis de la población y el establecimiento de los objetivos permite tomar medidas más directas y hacer diseños más realistas, orientado una evaluación del programa más objetiva (Becoña, 2002).

Los programas de prevención pueden estar centrados en diferentes ámbitos, siendo el escolar uno de los que más ha cobrado importancia, ya que se ha detectado una mayor afectación y el inicio del consumo más frecuente en este ámbito. La prevención escolar permite llegar a los jóvenes en la edad de mayor riesgo de inicio de consumo de distintas sustancias (De Vicenzi y Bareilles, 2011). 
Becoña (2002) establece principios para la estructuración de los programas en tema de prevención, entre los cuales están: basarse en las necesidades de la población y los factores de riesgo detectados; estructurar las exigencias metodológicas; orientarse al fortalecimiento de los principales factores de protección; basarse en la atención a todas las formas de abuso, incluidas las sustancias legales y socialmente aceptadas; incluir el control de la oferta, el acceso a las sustancias, desarrollo de habilidades para la vida; orientarse al manejo de los grupos de mutua ayuda como métodos interactivos entre los jóvenes; proyectarse a largo plazo, durante el año escolar, con varias intervenciones que permitan reforzar las metas de prevención; incluir la vinculación del contexto familiar y el manejo, tanto con el paciente, como con la familia; incluir trabajo de campañas comunitarias en las que se integra comunicación en masas y cambios en las políticas públicas; contar con un diseño diferencial de acuerdo a la edad y ser específicos para el grupo etario.

Los programas de prevención deben contar con una estructura lógica, donde tanto su contenido, como procedimientos de implementación, seguimiento y evaluación, tienen que partir de las necesidades concretas y delimitar el alcance y rango de acción prevista, asegurando un diseño acorde a la realidad de la población objeto.

Acción preventiva, por su lado, se refiere a los actividades y procedimientos concretos, específicos que permiten lograr los objetivos propuestos en los programas preventivos. Las acciones preventivas deben ser coherentes con el programa, del cual hacen parte, y con el plan de prevención a nivel general (Martínez, 2006).

\section{Algunos aspectos relevantes para los Programas de Prevención}

La construcción de programas de prevención puede ser orientada desde diferentes perspectivas.

En primer lugar es importante delimitar el ámbito de intervención al cual se dirige el programa. En este aspecto se puede distinguir los siguientes: individual, de pares, familiar, escolar, comunitario, social y laboral. La intervención en cada uno de estos ámbitos apunta a minimizar los factores de riesgo y fortalecer los factores protectores propios de cada ámbito. En este aspecto, las investigaciones previas en el tema indican la presencia de factores específicos para cada ámbito, como, por ejemplo, dificultades a nivel de formación de habilidades cognitivas superiores (Mariño, Castro y Torrado, 2012), baja autoestima, autoconcepto y autoeficacia (López-Torrecillas, Peralta, Muñoz-Rivas y Godoy, 2003; López-Torrecillas, Salazar, García-Retamero y Arias, 2015), 
deficiencias en el manejo de estrategias de afrontamiento y asertividad (Llorens Aleixandre, Perelló del Río y Palmer Pol, 2004; López-Torrecillas, Salazar, Rueda y Fernández, 2014), aceptación positiva frente al consumo (De la Villa Moral y Ovejero, 2005; Ortiz, Lopera, González y Klimenko, 2017), entre otros. A nivel de los pares se resalta como factor de riesgo, por ejemplo, un abaja percepción de riesgo de consumo y cultura de ocio basada en el consumo (SEDRONAR, 2017; Caravaca et al., 2015; Quintero, Ortega y Fuentes, 2015), presencia de amistades relacionadas con el consumo y formas específicas que adopta el consumo en grupos juveniles (Caravaca et al., 2015; Villarreal, Sánchez y Musitu, 2013), entre otros. A nivel familiar existen también muchos factores que deben ser trabajados con el fin de poder prevenir el consumo, entre los culés se puede mencionar, por ejemplo, presencia de problemas en la resolución de problemas y dificultades en la comunicación familiar (Cid-Monckton y Pedrao, 2011), baja cohesion familiar y deficiencias en las pautas de crianza (Musitu, Jiménez y Murgui, 2007; Rueda y Monreal, 2012), presencia de consumo en familia (Ramírez y Andrade 2005). En el ámbito escolar son variados los factores que pueden llevar a aumentar el riesgo de consumo entre los cuales se pueden mencionar las dificultades en el rendimiento escolar y relación con los docentes, acoso escolar y problemas en la adaptación escolar (Gómez Rodríguez, Gutiérrez Herrera y Londoño, 2013), entre otros. A nivel social y comunitario se resaltan aspectos como aceptación social de consumo (López y Rodríguez-Arias, 2010), promoción de consumo en medios de comunicación e influencia de redes sociales (Cruz-Juárez, Montero-Domínguez, Salas-García, Ortiz-León, 2016).

Otro de los aspectos relevantes es la población objeto de intervención. En la delimitación poblacional, a su vez, se consideran aspectos como edad evolutiva, contexto socioeconómico concreto, y las necesidades particulares de comunidad y/o grupo a intervenir.

En este orden de ideas, existen edades evolutivas con mayor vulnerabilidad hacia el consumo. Por ejemplo, los autores indican la importancia de la prevención en población adolescente, debido a la complejidad de los procesos psicológicos relacionados con esta y vulnerabilidad hacia las influencias sociales, razón por la cual la prevención en esta etapa genera una estimulación del desarrollo integral del adolescente, brindando la información y estrategias correctas que le permitan detectar y definir su postura frente al problema que se pretende evitar (Cepeda Diaz, Pezzano de Vengoechea y Racedo de Barranco, 1990).

Igualmente, es importante considerar el modelo de prevención que subyace a la construcción del programa. Por ejemplo, Pastor y López-Latorre (1993) 
exponen una propuesta de clasificación de los modelos teóricos como el médico, jurídico, distribución del consumo, sociológico, socio-cultural, psicosocial y ecológico, donde cada uno plantea su base metodológica y ofrece explicaciones sobre el origen, el desarrollo y el mantenimiento del consumo en las personas.

Los modelos de los cuales parte la orientación y planteamiento del programa promueven un conjunto de medidas específicas sobre cómo combinar e integrar distintos elementos a la hora de abordar la población objeto. Sin embargo, la misma naturaleza miltidimencional del fenómeno de consumo exige un abordaje integral de este desde una perspectiva multifocal, incidiendo sobre los diversos tipos de factores implicados en el origen del problema (Pastor y López-Latorre, 1993).

A nivel general existen varios principios orientadores para el proceso de planeación y diseño de programas de prevención. Entre estos se pueden resaltar los siguientes.

Apuntar a disminución de factores de riesgo y potenciación de factores protectores. Según Quintero Ordoñez, Ortega Carrillo y Fuentes Esparrell (2015), la construcción de los programas de prevención requiere de la información y la formación, el desarrollo de diversas habilidades para afrontar la presión e influencia social, estrategias para la toma de decisiones y estrategias que permitan fomentar los hábitos de vida saludables. Los programas deben ser duraderos y estables en el tiempo, se requiere una caracterización de la población, centrarse en acciones puntuales.

Orientarse a las características específicas de la población objeto de estudio, lo cual requiere uso de enfoque de prevención basada en evidencia, realizando estudio exploratorio y descriptivos previos, con el fin de definir bien tanto las características de consumo, como de los factores influyentes en este en la población objeto.

Apuntar a una atención integral tanto del sujeto como de su entorno familiar, educativo y social-comunitario. La vinculación de la familia y el entorno educativo debe ser prioridad para los programas preventivos, entrelazando el papel y la función de cada uno dentro de la vida del individuo. Atención a los ambientes familiares y educativos permite orientarse a todas las edades evolutivas, ya que la familia representa un factor protector desde el inicio de la vida del ser humano. Igualmente se facilita la prevención en momentos evolutivos transicionales de la vida, como de la escuela primaria a bachillerato, o estudios universitarios, etc. (Agudelo Bedoya y Estrada Arango, 2012). Los estudios indican que fomentar unos estilos educativos asertivos permite 
disminuir el riesgo de consumo de niños y adolescentes (Prieto-Montoya, Cardona-Castañeda, Vélez-Álvarez, 2016)

Participación y empoderamiento de la población objeto en el proceso de prevención. La participación activa de la población objeto permiten implementar con mayor eficacia prevención a nivel comunitario en diferentes ambientes: las escuelas, barrios, comunidades enteras y medios de comunicación, logrando na mayor consistencia y alcance de estrategias preventivas. La vinculación de la población objeto dentro de la estructuración del programa, permite generar mayor confianza en los demás; la vinculación con los objetivos y las metas, brinda un sentido de pertenencia con la causa y la finalidad de la prevención. Por ejemplo, la elaboración de campañas audiovisuales en la cual se cuente como protagonistas los mismos adolescentes, son una de las estrategias que se plantean para el abordaje de los programas hoy en día, donde el manejo de redes sociales y el desarrollo de las nuevas tecnologías son herramientas necesarias (Vidales y Rubio, 2015; Azorín Abellan, 2016).

Un larga duración y una evaluación continua del proceso. Por su complejidad, debido a una amplia variedad de factores y ámbitos de prevención, los programas deben contar con larga duración y constante evaluación, permitiendo su corrección y ajuste necesario durante el proceso. Esta última se puede realizar en varios momentos: inicial como un instrumento diagnóstico, de seguimiento y final. Algunos programas presentan el uso de una evaluación continuada, en la que evalúan los conocimientos de los participantes, rescatan la importancia de conocer los esquemas imaginarios del colectivo en referente a las sustancias (Azorín Abellan, 2016). La evaluación final permite la comparación de la información y la verificación de los nuevos conocimientos de los sujetos implicados, las nuevas ideas y los nuevos paradigmas que también pueden surgir con esto. Varios de los instrumentos utilizados son cuestionarios sobre drogas, observación participante y las fichas de trabajo (recogidas durante las sesiones grupales) (Azorín Abellan, 2016).

En cuanto a las experiencias del desarrollo de programas Colombianos, inicialmente la realidad de nuestro país es compleja, las necesidades de nuestro contexto son únicas y han dependido de evoluciones de factores como el político, el social, el económico y el cultural. El desarrollo de programas de prevención debido a los factores desencadenantes y mantenedores de la problemática, requieren el abordaje de los distintos tipos de prevención (Chaux, 2005).

En el análisis del contexto colombiano se rescata el abordaje de estrategias para la vida, sobre todo en cuanto a la agresión y la violencia, debido al aprendizaje que tenemos con respecto a nuestra historia. Considerando 
los aspectos particulares de la sociedad colombiana es necesario: fomentar el desarrollo de habilidades, competencias y valores, donde la transmisión de valores debe ser una práctica común y reforzada dentro de nuestros centros educativos; incluir a las familias y a los individuos en los programas de prevención, manejando un enfoque de atención primaria en salud mental con base en el desarrollo familiar y social; generar espacios de prevención temprana, en la que se busque el desarrollo de factores protectores desde los primeros ciclos de vida (Chaux, 2005).

Un proceso de prevención estructurada a nivel nación y continuo permite reducir costos de atención en salud mental en farmacodependencia (Pedraza, Molerio, Álvarez, Hernández y Rodríguez, 2012). Igualmente es necesario tener en cuenta que de la vinculación del ente territorial dentro de los programas de prevención, la caracterización de la población, la identificación de las necesidades, el manejo dentro del entorno educativo y el acceso a la población, requiere de la disponibilidad y uso de políticas que permitan el libre desarrollo de estas estrategias en las que se trabaje un tema de corresponsabilidad, enfocándose en la defensa de los derechos y prioridad de manejo del individuo. El establecimiento de políticas permite el uso y el acceso a presupuestos que apoyen el desarrollo de los programas, ya que económicamente requieren de un rubro específico para su accionar (Pedraza et al., 2012).

La buena estructuración de los programas parte, necesariamente, del análisis del contexto, la caracterización de la población, soporte de los elementos teóricos que sustenten los procesos de prevención, y adecuada planeación de las estrategias y ejercicios a utilizar. La prevención es un tema de bastante importancia y el planteamiento de los programas como pudimos observar requiere de interés, estudio y una buena estructuración. El consumo de sustancias es una problemática que hoy en día se encuentra en auge y cada vez son más los alcances de esta, las acciones de prevención deben existir y deben ser en disposición para toda la población, de acceso libre y de trabajo constante.

\section{Referencias}

Agudelo Bedoya, M., Estrada Arango, P. (2012). Cultura de prevención del consumo de sustancias psicoactivas (SPA) en la familia. Trabajo Social, 14: 79-92, Recuperado desde https://revistas.unal.edu.co/index.php/tsocial/article/view/37257

Azorín Abellan, C. M. (2016). iAbre los ojos! un proyecto de mejora educativa para la prevencion de drogas en adolescentes. Revista complutense de educación, 27(1): 141-159. http://revistas.ucm.es/index.php/RCED/article/view/45532 
Becoña Iglesias, E. (2001). Bases teoricas que sustentan los programas de prevencion. Madrid: Plan nacional de drogas de España. Universidad Santiago de Compostela.

Becoña Iglesias, E. (2002). Bases Científicas de la Prevención. Madrid: Delegación del gobierno para el Plan Nacional sobre Drogas.

Botvin, G., Epstein, J., Baker, E., Diaz, T., Ifill-Williams, M., Miller, N., \& Cardwell, J. (1997). Schoolbased drug abuse prevention with inner-city minority youth. Journal of Child and Adolescent Substance Abuse, 6: 5-19, http://citeseerx.ist.psu. edu/viewdoc/download?doi=10.1.1.522.5556\&rep=rep1\&type $=$ pdf

Burkhart, G. (2011). Environmental drug prevention in the EU. Why is it so unpopular? Adicciones, 23 (2): 87-100, http://www.prevencionbasadaenlaevidencia.net/ uploads/PDF/RP_Prevencion_ambiental_drogas_Burkhart.pdf

Castaño, G. (2006). Nuevas tendencias en prevención de las drogodependencias. Revista Salud y Drogas, 6(2): 127-148. Recuperado desde http://www.redalyc. org/html/839/83960202/

Castellanos Obregón, J. M., \& Espinosa Herrera, G. (2013). Revision de las tendencias de investigacion sobre consumo de sustancias ilegales por los jovenes. Revista de Antropología y Sociología: Virajes, 15 (2): 57-71, Recuperado desde http://vip. ucaldas.edu.co/virajes/downloads/Virajes15(2)_3.pdf

Caravaca, J., Noh, S., Hamilton, H., Brands, B., Gastaldo, D., Miotto Wriget, M. (2015). Factores socioculturales y consumo de drogas entre estudiantes universitarios costarricenses. Texto Contexto Enferm, Florianópolis, 24 (Esp): 145-53, http:// dx.doi.org/10.1590/0104-07072015001170014

Cepeda Diaz, J., Pezzano de Vengoechea, G., \& Racedo de Barranco, M. (1990). Fundamentos teoricos para desarrollar programas de prevencion primaria de la farmacodependencia en las distintas etapas del ciclo evolutivo. Revista Investigacion \& Desarrollo Universidad del Norte, 1(1): 93-113, Recuperado desde http://ciruelo.uninorte.edu.co/pdf/invest_desarrollo/1/6\%20Fundamentos\%20 teoricos\%20para\%20desarrollar\%20programas\%20de\%20prevenci\%F3n.pdf

Cid-Monckton, P., Pedrao, L. (2011). Factores familiares protectores y de riesgo relacionados al consumo de drogas en adolescentes. Rev. Latino-Am. Enfermagem, 19:738-745, http://dx.doi.org/10.1590/S0104-11692011000700011

Cogollo, Z. (2013). La prevencion del inicio del consumo de cigarrillo en escolares: Una mirada critica a los estudios basados en la escuela. Revista de salud publica, 15(2): 307-315. Recuperado desde https://revistas.unal.edu.co/index.php/ revsaludpublica/article/view/37630/62092 
Correa Muñoz, A., Perez Gomez, A. (2013). Relacion e impacto del consumo de susutancias psicoactivas sobre la salud en Colombia. Liberabit, 19(2): 153162. Recuperado desde http://www.scielo.org.pe/scielo.php?script=sci arttext\&pid $=$ S1729-48272013000200001\&lng =es\&tlng =es

Chaux, E. (2005). El Programa de Prevención de Montreal: Lecciones para Colombia. Revista de Estudios Sociales, 21: 11-25. Recuperado desde https://revistas. uniandes.edu.co/doi/abs/10.7440/res21.2005.01

Cruz-Juárez, A., Montero-Domínguez, F., Salas-García, B., Ortiz-León, C. (2016). Redes sociales y su influencia en el consumo de drogas en estudiantes de preparatoria en Xalapa, Veracruz: Un estudio cualitativo. Revista Investigaciones Sociales, 2 (6): 14-22, Recuperado desde http://www.ecorfan.org/ republicofnicaragua/researchjournal/investigacionessociales/journal/vol2num6/ Revista_Investigaciones_Sociales_V2_N6_2.pdf

De la Villa Moral, M., Ovejero, A. (2005). Modificación de las actitudes, hábitos y frecuencia de consumo de alcohol y otras sustancias psicoactivas en adolescentes españoles a partir de un programa educativo-preventivo. Revista Colombiana de Psicología, 14: 100-118, Recuperado desde http://bdigital.unal.edu. co/13659/1/1224-6112-1-PB.pdf

De Vicenzi, A., Bareilles, G. (2011). Promoción de la salud y prevención escolar del consumo de drogas en contextos de vulnerabilidad social. Educacion y Educadores, 14 (3): 577-600. Recuperado desde http://educacionyeducadores.unisabana.edu. co/index.php/eye/article/view/2047/2600

Fernandez Cruz, M., Gijón Puerta, J., Lizarte Simon, E. (2016). Consumo de sustancias psicoactivo en estudiantes de magisterios y sus creencias acerca de la educación preventiva. Accion Psicologica, 13 (1): 67-78, http://dx.doi.org/10.5944/ ap.13.1.17419

Ferrel Ortega, F. R., Ferrel Ballestas, L. F., Alarcon , B. A., Delgado Arrieta, K. D. (2016). Consumo de Sustancias psicoactivas como indicador de deterioro de la salud mental en jovenes escolarizados. Psychologia: Avances de la disciplina, 10(2): 43-54, Recuperado desde http://www.scielo.org.co/pdf/psych/v10n2/v10n2a04. pdf.

Galvan, G., Guerrero Martelo, M., Torres Oviedo, J., Torres, F., Sanchez Caraballo, J. (2015). Prevalencia de uso de cannabis: perfil familiar y social de una muestra adolescente. Psychologia: Avances de la disciplina, 9(2): 101-112, Recuperado desde http://www.scielo.org.co/pdf/psych/v9n2/v9n2a08.pdf

Gómez Rodríguez, D., Gutiérrez Herrera, M., Londoño, S. (2013). Depresión y consumo de sustancias psicoactivas en adolescentes en la ciudad de Bogotá. 
Psychologia, Avances de la disciplina, 7 (1): 45-51. Disponible en: http://www. scielo.org.co/pdf/psych/v7n1/v7n1a05.pdf

Hernández Ramírez, E. M. (2015). Evaluacion de un programa de prevencion del consumo de sustancias psicoactivas para la infancia. Salud y drogas, 15(1): 67-77 Recuperado desde http://www.redalyc.org/articulo.oa?id=83938758007.

López, S., Rodríguez-Arias, J. (2010). Factores de riesgo y de protección en el consumo de drogas en adolescentes y diferencias según edad y sexo. Psicothema, 22 (4): 568-573, Recuperado desde www.psicothema.com

López-Torrecillas, F., Peralta, I., Muñoz-Rivas, M. J., Godoy, J. F. (2003). Autocontrol y consumo de drogas. Adicciones, 15(2):127-136. http://www.adicciones.es/ index.php/adicciones/article/view/436

López-Torrecillas, F., Salazar, I. C., Rueda, M. M., Fernández, E. C. (2014). La capacidad predictiva de la asertividad en las recaídas de los drogodependientes. Psicología Conductual, 22(3), 603-616, Recuperado desde https://web.b.ebscohost.com/ abstract ?direct $=$ true $\&$ profile $=$ ehost $\&$ scope $=$ site \&authtype

López-Torrecillas, F., Salazar, I. C., García-Retamero, R., Arias, R. M. (2015). Autoeficacia de personas drogodependientes en tratamiento. Psicología Conductual, 23(2), 325-343, Recueprado desde http://psycnet.apa.org/ record/2015-41487-007

Llorens Aleixandre, N., Perelló del Río, M., Palmer Pol, A. (2004). Estrategias de afrontamiento: factores de protección en el consumo de alcohol, tabaco y cannabis. Adicciones, 16 (4): 1-6, http://www.adicciones.es/index.php/adicciones/article/ view/391

Martínez, E. (2006). Hacia una Prevención con Sentido. Bases del centro de prevención e investigación de la fundación colectivo aquí y ahora. Bogotá: Colectivo Aquí y Ahora.

Mariño, N., Castro, J., Torrado, J. (2012). Funcionamiento ejecutivo en policonsumidores de sustancias psicoactivas. Revista de Psicología Universidad de Antioquia, 4 (2), 49-64, Disponible en https://dialnet.unirioja.es/descarga/ articulo/4865179.pd

Ministerio de la Protección Social, República de Colombia. (2011). Manual para la detección e intervención temprana del consumo de sustancias psicoactivas y problemas de salud mental en los servicios de salud amigables para adolescentes y jóvenes. Documento anexo al manual de servicios amigables. Bogotá: Alvi Impresores. Recuperado desde http://proinapsa.uis.edu.co/redcups/Biblioteca/ Consumos\%20Nocivos/manual_deteccion_consumos.pdf 
Musitu, G., Jiménez, T., Murgui, S. (2007). Funcionamiento familiar, autoestima y consumo de sustancias en adolescentes: un modelo de mediación. Salud Pública de México, 49(1), 3-10. Disponible en http://www.scielo.org.mx/scielo. php?script $=$ sci_arttext\&pid=S0036-36342007000100002\&lng=es\&tlng =es.

Muñoz Ortega, L. M., Barbosa R, L. C., Briñez, J. A., Caycedo, C. C., Mendez Heilman, M., \& Oyuela Vargas, R. (2012). Elemenos para programas de prevencion en consumo de alcohol en universitarios en Bogotá, Colombia. Universitas Psychologica, 11 (1): 131-145, Recuperado desde http://revistas.javeriana.edu. co/index.php/revPsycho/\%20article/view/602.

NIDA (Instituto Nacional sobre el abuso de Drogas) (2004). Cómo prevenir el uso de drogas en los niños y los adolescentes. Departamento de salud y servicios humanos de los Estados Unidos. Recuperado desde https://www.drugabuse.gov/sites/default/ files/redbook_spanish.pdf

ODC (Observatorio de drogas en Colombia) (2017). Reporte de drogas de Colombia. Gobierno de Colombia. Disponible en http://www.odc.gov.co/Portals/1/ publicaciones/pdf/odc-libro-blanco/reporte_drogas_colombia_2017.pdf

Ortiz, J., Lopera, N., González, N., Klimenko, O. (2017). Actitudes de favorabilidad hacia conductas adictivas prevalentes en una muestra de adolescentes entre 12 y 18 años de algunas Instituciones Educativas en Medellín y Ríosucio. Revista Psicoespacios, 11(19): 24-45, https://doi.org/10.25057/issn.2145-2776

Pastor, J., López-Latorre, M. (1993). Modelos teóricos de prevención en toxicomanías: una propuesta de clasificación. Anales de Psicología, 9(1), 19-30, Recuperado desde http://www.um.es/analesps/v09/v09_1/03-09_1.pdf

Pedraza, L., Molerio, O., Álvarez, R., Hernández, Y., Rodríguez, Y. (2012). Prevención y tratamiento del tabaquismo desde un enfoque psicológico. Psicogente, 15(28), 445-461, Recuperado desde http://publicaciones.unisimonbolivar.edu.co/ rdigital/ojs/index.php/psicogente/article/view/374/0

Petticrew, M., Roberts, H. (2008). Systematic reviews in the social sciences: A practical guide. John Wiley \& Sons. DOI:10.1002/9780470754887

Prieto-Montoya, J., Cardona-Castañeda, L. M., Vélez-Álvarez, C. (2016). Estilos parentales y consumo de sustancias psicoactivas en estudiantes de $8^{\circ}$ a $10^{\circ}$. Revista Latinoamericana De Ciencias Sociales, Niñez y Juventud, 14(2), 13451356, Recuperado desde http://www.scielo.org.co/pdf/rlcs/v14n2/v14n2a32. pdf

Moreno, O., Posada, I., Jaramillo, A. (2012). Analisis de proyectos gestionados para educadores: El reto de la prevencion del consumo de sustancias psicoactivas y violencias en jovenes en Medellín. Revista de la facultad nacional de salud publica, 
30(3): 273-281, Recuperado desde http://aprendeenlinea.udea.edu.co/revistas/ index.php/fnsp/article/view/11365

Quintero Ordoñez, B., Ortega Carrillo, J., \& Fuentes Esparrell, J. A. (2015). Eficacia del programa de prevencion de adicciones Entre-Todos (Proyecto Hombre). Revista Lasallista de investigación, 12 (2): 28-35, Recuperado desde http://www. redalyc.org/pdf/695/69542291004.pdf.

Ramírez, M., Andrade D. (2005). La familia y los factores de riesgo relacionados con el consumo de alcohol y tabaco en los niños y adolescentes (Guayaquil-Ecuador). Rev Latino-am Enfermagem, 13:813-8, Recuperado desde http://www.scielo.br/ pdf/rlae/v13nspe/v13nspea08.pdf

Ruiz Olivares, R., Lucena, V., Jose Pino, M., Raya, A., Herruzo, J. (2010). El consumo de cannabis y la percepcion del riesgo en jovenes universitarios. Behavioral Psychology, 18 (3): 579-590, Recuperado desde https://www.researchgate.net/ publication/259602406_EL_CONSUMO_DE_CANNABIS_Y_LA_PERCEPCION_ DEL_RIESGO_EN_JOVENES_UNIVERSITARIOS_1

Rueda, E., Monreal, M. (2012). Consumo de sustancias en la adolescencia: un análisis de las diferencias desde el punto de vista psicosocial. IV Congreso Universitario Nacional Investigación y Género, Ministerio de Educación y Ciencia de España. Disponible en https://idus.us.es/xmlui/bitstream/handle/11441/40667/Pages\%20from\%20 Investigacion_Genero_12-1509-2188-5.pdf? sequence $=1$ \&isAllowed $=\mathrm{y}$

Sáenz Uribe, S. (2009). Consumo de drogas ilictas, ¿prohibicion o regulacion? Analisis del caso colombiano en perspectiva de politica comparada. Facultad de derecho y ciencias politicas, 39 (111): 253-282, Recuperado desde https://revistas.upb.edu. co/index.php/derecho/article/view/660

SEDRONAR (Secretaria de Políticas Integrales sobre drogas de Nación Argentina) (2017). Estudio nacional en población de 12 a 65 años sobre consumo de sustancias psicoactivas. Argentina, 2017. Factores de riesgo en el consumo de sustancias psicoactivas. Recuperado desde http://www.observatorio.gov.ar/media/k2/ attachments/FactoresZdeZRiesgoZZ2017ZZ3Zenero.pdf

UNODC (Oficina de las Naciones Unidas contra la droga y el delito). (2015). Infome Mundial sobre Drogas. Recuperado desde https://www.unodc.org/documents/ wdr2015/WDR15_ExSum_S.pdf

UNODC (Oficina de las naciones unidas contra la droga y el delito) (2016). Informe mundial sobre las drogas. Recuperado desde https://www.unodc.org/doc/ wdr2016/WDR_2016_ExSum_spanish.pdf

Vidales, N. L., Rubio, L. G. (2015). Análisis y proyección de los contenidos audiovisuales sobre jóvenes y drogas en YouTube. Estudios Sobre El Mensaje Periodístico, 21 (2): 
863-881. Recuperado desde http://revistas.ucm.es/index.php/ESMP/article/ view/50889

Villarreal, M. Sánchez, J., Musitu, G. (2013). Análisis psicosocial del consumo de alcohol en adolescentes mexicanos. Universitas Psychologica, 12(3): 857-873, doi:10.11144/Javeriana.UPSY12-3.apca 